\title{
"Identification of potential biomarkers associated with pathogenesis of primary prostate cancer based on meta-analysis approaches".
}

\author{
Neda Sepahi ${ }^{1,2}$, Mehrdad Piran $^{1,2}$, , Mehran Piran ${ }^{1,3}$, Ali Ghanbariasad ${ }^{1,2^{*}}$ \\ ${ }^{1}$ Department of Medical Biotechnology, Fasa University of Medical Sciences, Fasa, Iran \\ ${ }^{2}$ Noncommunicable Diseases Research Center, Fasa University of medical sciences, Fasa, Iran \\ ${ }^{3}$ Bioinformatics and Computational Biology Research Center, Shiraz University of Medical Sciences, Shiraz, Iran
}

\begin{abstract}
Worldwide prostate cancer (PCa) is recognized as the second most common diagnosed cancer and the fifth leading cause of cancer death among men globally. Rising incidence rates of PCa have been observed over the last few decades. It is necessary to improve prostate cancer detection, diagnosis, treatment and survival .However, there are few reliable biomarkers for early prostate cancer diagnosis and prognosis. In the current study, systems biology method was applied for transcriptomic data analysis to identify potential biomarkers for primary PCa. We firstly identified differentially expressed genes (DEGs) between primary PCa and normal samples. Then the DEGs were mapped in Wikipathways and gene ontology database to conduct functional categories enrichment analysis. 1575 unique DEGs with adjusted p-value $<0.05$ were achieved from two sets of DEGs. 132 common DEGs between two sets of DEGs were retrieved. The final DEGs were selected from 60 common upregulated and 72 common downregulated genes between datasets. In conclusion, we demonstrated some potential biomarkers (FOXA1, AGR2, EPCAM, CLDN3, ERBB3, GDF15, FHL1, NPY, DPP4, and GADD45A) and HIST2H2BE as a candidate one which are tightly correlated with the pathogenesis of PCa.
\end{abstract}

\section{Introduction}

Worldwide prostate cancer $(\mathrm{PCa})$ is recognized as the second most common diagnosed cancer and the fifth cause of cancer death among men globally, with an estimated 1.1 million global number of new cases diagnosed and 307,000 deaths in 2012 (1). The most important risk factors associated with PCa include age, family history, genetic factors, lifestyle, environmental influences, and diet. Rising incidence rates of PCa have been observed over the last few 
decades, largely due to screening and early detection procedures (2). Studies based on analyzing Nordic twin registries have demonstrated at least a 50\% higher risk in monozygotic twins than dizygotic twins, suggesting that genetic rather than shared lifestyle factors are responsible for much of this familial aggregation (3, 4). Indeed, genetic factors have been estimated to be responsible for almost $42 \%$ of the risk (4). Cell survival and proliferation, acquired through deregulation of the cell cycle, are prerequisites for cancer (5, 6). The key molecules that regulate the cell cycle include cyclin-dependent kinases (CDKs) and their regulatory cyclin partners. Regulation of these complexes involves the control of cyclin production and its destruction through phosphorylation by specific kinases and other regulatory proteins $(7,8)$. Identification of potential and reliable biomarkers which are involved in the cell cycle and proliferation processes can be beneficial for early PCa detection, diagnosis and prognosis.

Over recent years, considerable researches have led to the development of several molecular and genetic assays that have provided a prospective direction for the development of prostate cancer biomarkers (9). High-throughput genomics technologies (e.g., gene expression microarrays) have been significantly changing biomedical research nowadays, which the expression patterns of thousands of genes can be monitored simultaneously (10). The emergence of novel high throughput technologies make the rapid identification of a single or groups of biomarkers possible $(11,12)$. Identification of differentially expressed genes (DEGs) often leads to the identification of novel cancer biomarkers followed by detecting upstream cancer causal genes by subsequent network analysis (13). In the current study, we downloaded two transcriptomic datasets from the Gene Expression Omnibus (GEO) in order to systematically detect DEGs. We constructed protein-protein interaction (PPI) network of the DEGs. Then, the gene ontology and Wikipathway of DEGs were done for the enrichment part. To get this goal, computational systems biology were applied to obtain a number of primary PCa DEGs from the results of transcriptomic analysis. Then, our findings may reveal the potential biomarkers in primary $\mathrm{PCa}$ detection and diagnosis.

\section{Methods}

\section{Database Searching and recognizing pertinent experiments}

From Gene Expression Omnibus (http://www.ncbi.nlm.nih.gov/geo/) database (GEO), we downloaded two PCa-related microarray datasets containing high quality transcriptomic samples concordance to the study design which compared the transcriptome of patients with 
the normal individuals. Searches were filtered for Homo sapiens and prostate cancer (PCa), benign and primary were the key words utilized in the search. Microarray raw data with accession numbers GSE6604, GSE6606 and GSE69223 were downloaded from this database. GSE69223 dataset (first dataset) which compared the transcriptome of 15 prospectively collected. It contained 15 benign PCa tumor biopsies and 15 normal samples from adjacent primary PCa tumors. GSE6604 included normal samples and GSE6606 contains primary PCa samples. So, we merged two datasets into a unified dataset (GSE6604GSE6606/Second dataset) consisting of 18 normal samples and 61 primary samples. All the samples ID are listed in supplementary file 1.

\section{Meta-Analysis and Identifying Differential Expressed Genes}

$\mathrm{R}$ software was utilized to import and analyze data for each dataset separately. Two main Preprocessing steps involving background correction and probe summarization were performed using RMA method in Affy package (14). Absent probesets were also identified using "mas5calls" function in this package. For each probeset half of the samples had absent values, that probeset was regarded as absent and removed from the expression matrix. Besides, Outlier samples were identified and removed using PCA and hierarchical clustering approaches. Next, data normalization was done via quantile normalization method (15). Then, standard deviation (SD) for each gene was computed and SDs median was used as a cut off to remove low variant genes. NsFilter function in genefilter package was used for solving many to many problem (16) which is mapping multiple probesets to the same gene symbol (17). This function selects the probeset with the highest Interquartile range (IQR) between multiple probesets mapping to the same gene symbol. Limma package in $\mathrm{R}$ software was applied to perform linear models on the expression matrix data of each GEO dataset, and the DEGs between tumor and normal tissues were also screened by the limma package (18). $|\log 2 \mathrm{FC}|>1$, Benjamini Hochberg adjusted P-value 0.05 (6) were considered statistically significant for the DEGs. Finally, common DEGs between two datasets were selected as the final DEGs.

\section{Network Construction}

The STRING database was applied to identify potential interactions among the overlapping DEGs. The giant component of the weighted network was extracted from the whole network through using igraph package in R software (19). Next, the weighted adjacency matrix was transformed into a symmetric matrix to get modified into a new adjacency matrix using topological overlapping measure (TOM) function in WGCNA R package (20). To remain with a distance matrix, the new adjacency matrix was subtracted from one. 


\section{Neighbourhood Ranking to the Core Genes}

Using $\mathrm{R}$ software, a matrix of all shortest paths between all pairs of nodes in a weighted network was constructed using Dijkstra algorithm (21). Then, a distance score, Dj, for each node in the PPI network was calculated. $\mathrm{Dj}$ is the average of the shortest paths from all the noncore genes to reach the node $\mathrm{j}$ subtracted from the average of the shortest paths from the core genes to reach the node $\mathrm{j}$ normalized by the average of the all shortest paths to reach the node $\mathrm{j}$ from the whole network (22).

$$
D_{j}=\frac{\frac{\sum_{i \notin S} S P_{i j}}{|N S|}-\frac{\sum_{i \in S} S P_{i j}}{|S|}}{\frac{\sum_{i} S P_{i j}}{|S|+|N S|}}
$$

Formula1

This scoring system implies how close each node is to the core nodes. Here, $\mathrm{S}$ is the set of core nodes and also NS is the set of non-core nodes. As a result, a score bigger than zero implicates that node $\mathrm{j}$ falls closer on average to the core nodes than it does on average to the rest of the network. Nodes with positive score were kept and the remained ones were deleted from the network. D scores were computed without imposing any threshold on edge weights.

\section{Enrichment Analysis}

To explore the functions and pathways of DEGs, we carried out enrichment analysis. Enrichment analysis was performed using Enrichr online tool (23). Enriched terms for biological process were obtained from $G O$ repository. For pathway enrichment analysis, DEGs were mapped into the wikiPathways signaling repository version 2019 for human. Enriched terms with the top score and the p-value less than 0.05 were selected. 


\section{Results}

\section{Data Preprocessing}

Each dataset was imported into R separately. Almost $50 \%$ of probesets were regarded as absent and left out from the expression matrix to avoid technical errors to be engaged in downstream analysis. To be more precise in preprocessing step outlier sample detection was conducted using PCA and hierarchical clustering. Figure1A illustrates the PCA plot for the samples in first (GSE69223) dataset. The same plot was created for the samples in second (GSE6605GSE6606) study. Between two groups of samples, a number of them were away from their sets and should be considered as outliers. To be more specific, a hierarchical clustering method introduced by Oldham MC, et al (24) were used. Pearson correlation coefficients between samples was subtracted from one for measurement of the distances. Figure 1B depicts the dendrogram for the normal samples. Figure1C normal samples are plotted based on their Number-SD scores. To get this number for each sample, the average of whole distances was subtracted from distances average in all samples, then results of these subtractions were normalized (divided) by the standard deviation of distance averages (24). Samples with Number-SD less than negative two which were in a pretty long distance from their cluster set in the PCA plot, were regarded as outlier. Moreover, for every dataset, box plots were plotted and samples with extreme IQRs were removed. For instance, GSM1695600 was regarded as outlier based on the PCA and boxplot while GSM1695586 was considered as outlier based on Number-SD score. However, boxplot alone for some samples was sufficient to consider them as outliers regardless of PCA or Number-SD plots. Six outlier samples in first dataset and 31 outlier samples in second dataset were recognized. Supplementary file1 contains information about groups of samples and outliers. 


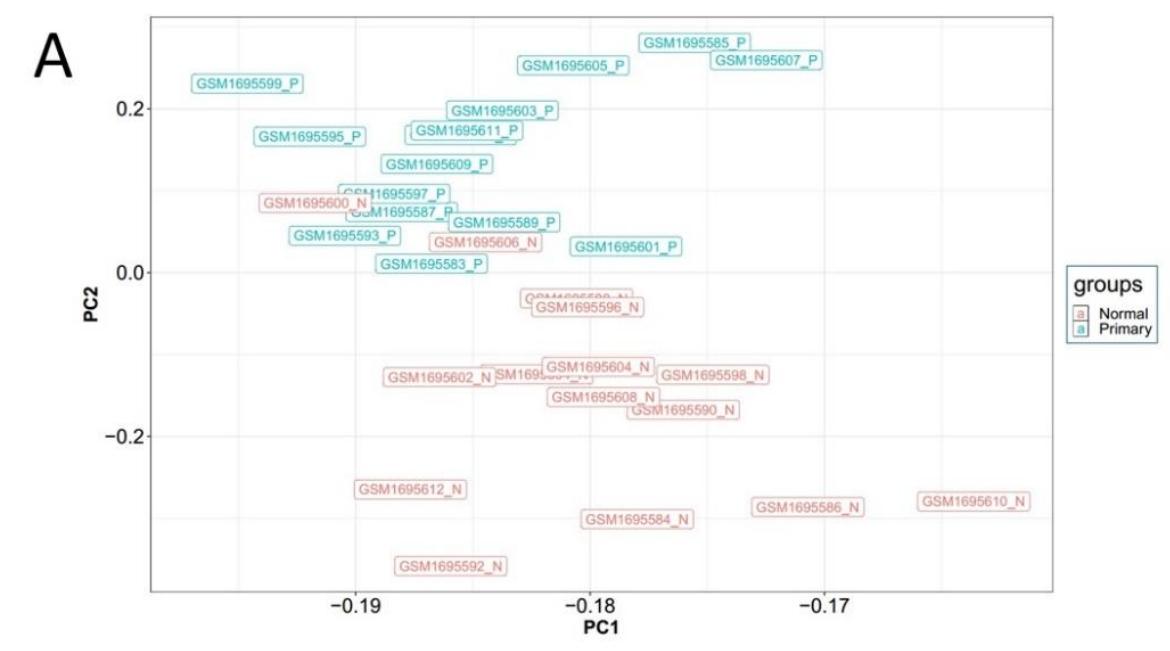

B
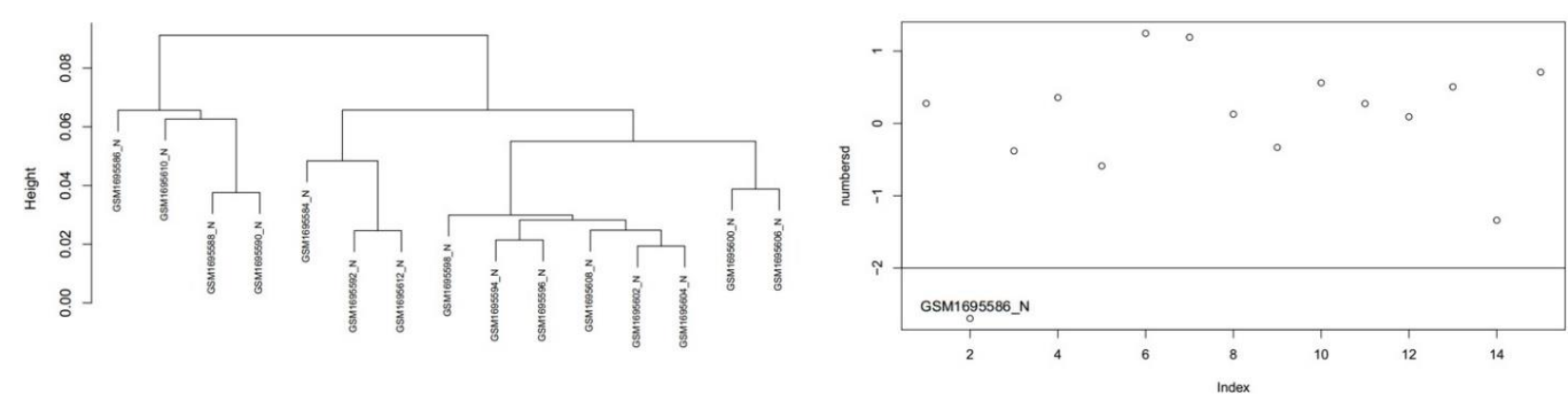

Figure 1: Illustration of outlier samples in first dataset. A, is the PCA plot, B is the dendrogram for the normal samples and $\mathrm{C}$ is the Number-SD plot for normal samples.

\section{Identifying Differentially Expressed Genes}

All the samples in two groups including primary and normal were put into the calculation of DEGs. Genes with absolute log fold change (LogFC) larger than 1 and adjusted p-value less than 0.05 were regarded as DEGs. All 268 samples were put into the calculation of DEGs. Totally, 504 upregulated genes and 909 down-regulated genes were recognized in the first dataset as well as 154 overexpressed genes and 145 down-regulated genes in the second dataset. The final DEGs were selected from 60 common upregulated and 72 common down-regulated genes between datasets. DEGs with the absolute LogFC larger than $90 \%$ quantile of all DEGs (final DEGs) were considered as the core genes. This threshold was 1.919744 for up-regulated and -1.998201 for down-regulated genes in the first dataset and 3.22436 for up-regulated and -2.37239 for down-regulated genes in the second dataset. The core genes are shown in the scatter plots in Figure 2. Housekeeping genes are situated on the diagonal of the plot whilst upregulated DEGs are above the diagonal and down-regulated DEGs are under the diagonal. 


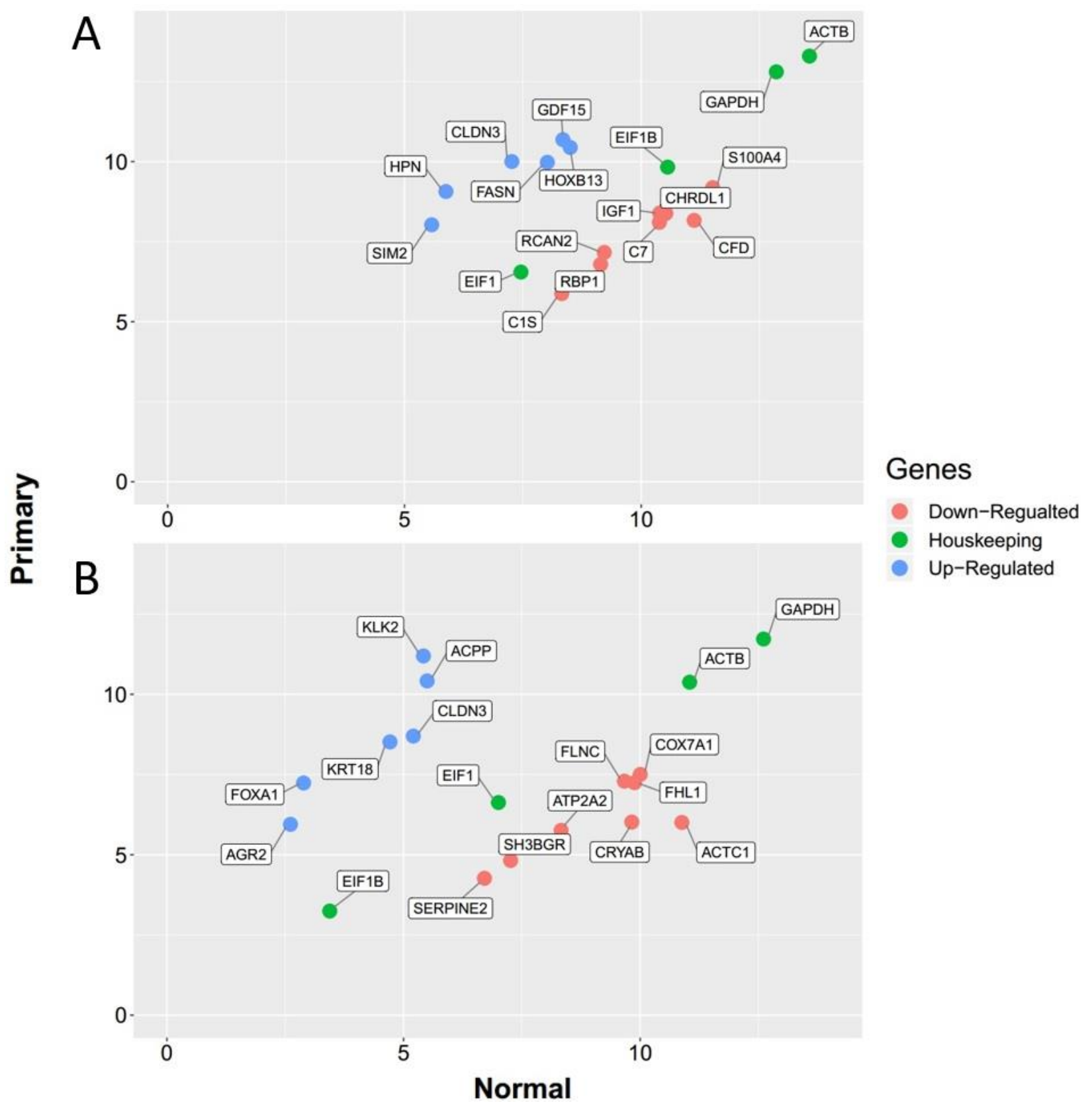

Figure2: The quality Control plot. The housekeeping genes are near the diameter of plot presenting close average values between three groups.

\section{Undirected Protein-Protein Interaction Network}

1575 unique DEGs with adjusted p-value $<0.05$ and absolute log fold change $>1$ were achieved from two sets of DEGs. Primary samples were compared to normal samples in both datasets. In the next step, 132 common DEGs between two sets of DEGs were retrieved. All DEGs for each dataset are presented in Supplementary file2. Common ones were employed to construct the Protein-Protein-Interaction (PPI) network. STRING database was used to 
bioRxiv preprint doi: https://doi.org/10.1101/2020.03.05.978205; this version posted March 5, 2020. The copyright holder for this preprint (which was not certified by peer review) is the author/funder. All rights reserved. No reuse allowed without permission.

generate the interactions based on seven filtrations namely, Neighborhood, Text mining, Experiments, Databases, Co-expression, Gene fusion, and Co-occurrence. STRING combined scores were used as the edge weights. This network involved 132 nodes and 248 edges. The giant component of this network with 97 nodes and 243 edges is presented in figure 3.

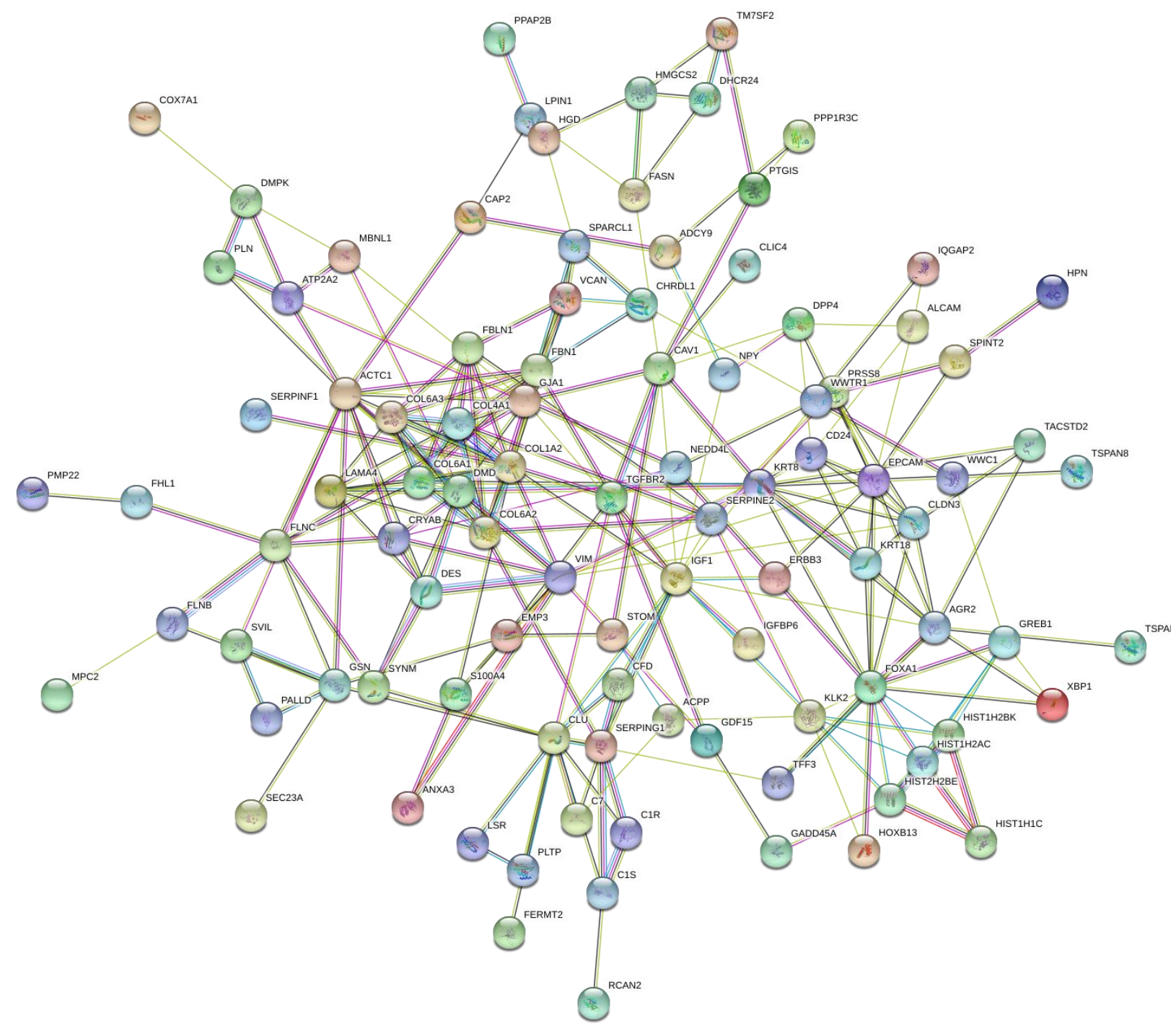

Figure3: The whole network giant component. Labels are protein/gene symbols and edges with stronger evidence are thicker. This is a scale free network (25) which follows a power law distribution (most of the network nodes have a low degree while there are few nodes with high degree). 


\section{Determination of Core Genes Neighborhood through Shortest Path-Based Scoring System}

In this step, we used weights between nodes as the estimation of distances in the weighted adjacency matrix. Nodes with shorter distances from the core genes were selected and a smaller network was extracted from the main network. In order to choose core genes, 6 common DEGs in the first quartile of the most upregulated or downregulated genes in two datasets were considered. Computing the shortest path score for the non-core genes, led to a network with 24 nodes consisting of 6 core nodes and 22 neighbors. This two-component graph is called Core network shown in figure 4A. The expression states for these genes are illustrated in Figure 4B. In addition, two important centralities including degree and betweenness for Core network nodes are depicted in figure 4B. FOXA1 one of the core genes has the highest value in both centralities. ARG2 and EPCAM have pretty high degree and betweenness centralities. HIST2H2BE and DPP4 have relatively high betweenness value.
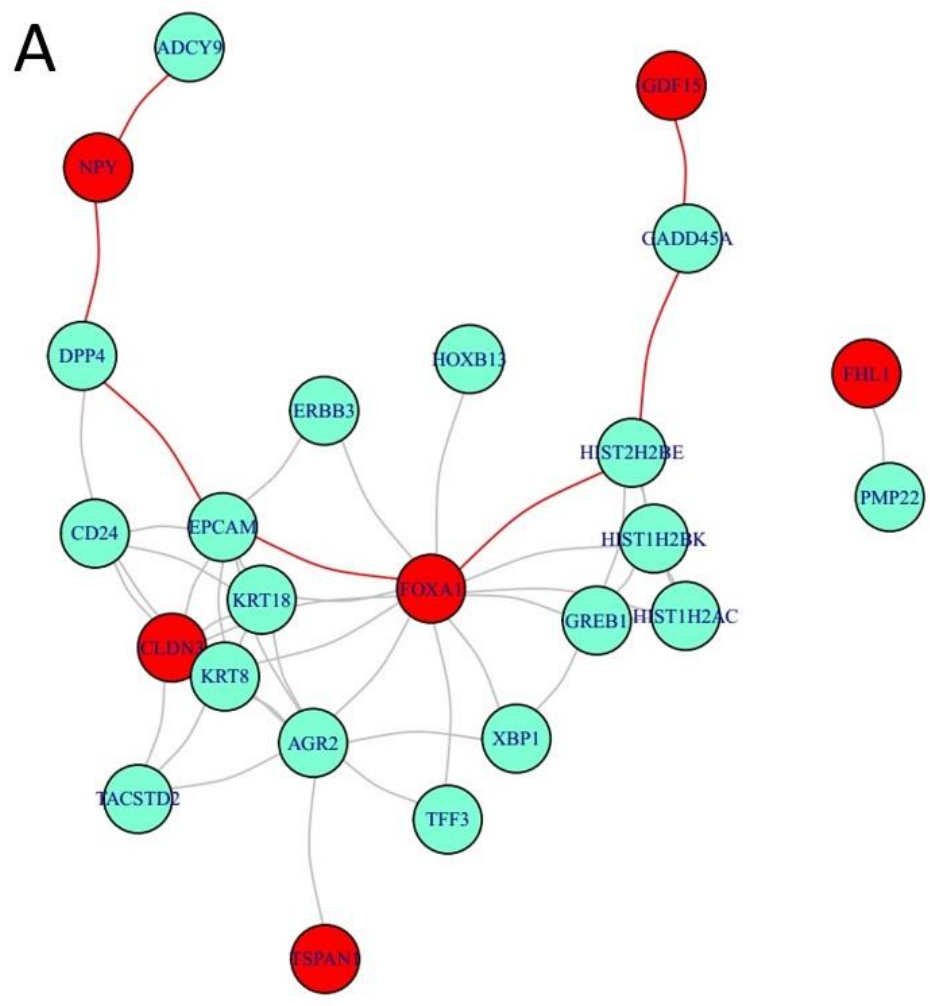

\begin{tabular}{|c|c|c|c|}
\hline DEGs & Degree & Betweenness & Expression \\
\hline FOXA1 & 13 & 112.75 & Up \\
\hline AGR2 & 9 & 34.93 & Up \\
\hline EPCAM & 8 & 52.3 & Up \\
\hline CLDN3 & 7 & 8.28 & Up \\
\hline KRT8 & 7 & 8.28 & Up \\
\hline KRT18 & 6 & 3 & Up \\
\hline HIST2H2BE & 5 & 38 & Up \\
\hline GREB1 & 5 & 2.5 & Up \\
\hline CD24 & 5 & 5.7 & Up \\
\hline HIST1H2AC & 4 & 0 & Up \\
\hline HIST1H2BK & 4 & 0 & Up \\
\hline DPP4 & 3 & 38 & Up \\
\hline XBP1 & 3 & 1.25 & Up \\
\hline TACSTD2 & 3 & 0 & Up \\
\hline NPY & 2 & 20 & Up \\
\hline TFF3 & 2 & 0 & Up \\
\hline ERBB3 & 2 & 0 & Up \\
\hline GADD45A & 2 & 20 & Down \\
\hline GDF15 & 1 & 0 & Up \\
\hline FHL1 & 1 & 0 & Down \\
\hline TSPAN1 & 1 & 0 & Up \\
\hline HOXB13 & 1 & 0 & Up \\
\hline PMP22 & 1 & 0 & Down \\
\hline ADCY9 & 1 & 0 & Down \\
\hline
\end{tabular}

Figure4: The Core network and centralities. This network presented in A contains two components and core genes are in red. B, Illustrates the Degree and Betweenness centralities for the nodes in the Core network. DEGs are sorted based on the highest Degree. 


\section{Network Descriptive}

The Core network diameter is seven containing GDF15, GADD45A, HIST2H2BE, FOXA1, EPCAM, DPP4, NPY, and ADCY9. The giant component descriptive is explained in the following. Transitivity was around 52\%, edge density was about $17.4 \%$ and the mean distance is 2.35. Other centralities for the nodes and the average distances between each node and the other nodes are provided in Supplementary file3.

\section{Network Clustering and Enrichment Analysis}

Giant component (GC) of Core network were clustered using fast greedy algorithm in R. It is illustrated in Figure 5. Gene Set Enrichment Analysis (GSEA) was performed on each cluster separately. Gene sets in clusters 1 to 3 were given to Enrichr online tool. Tables 2A and 2B depict the enrichment analysis for the core network giant component. Enriched terms are sorted based on the highest p-value.

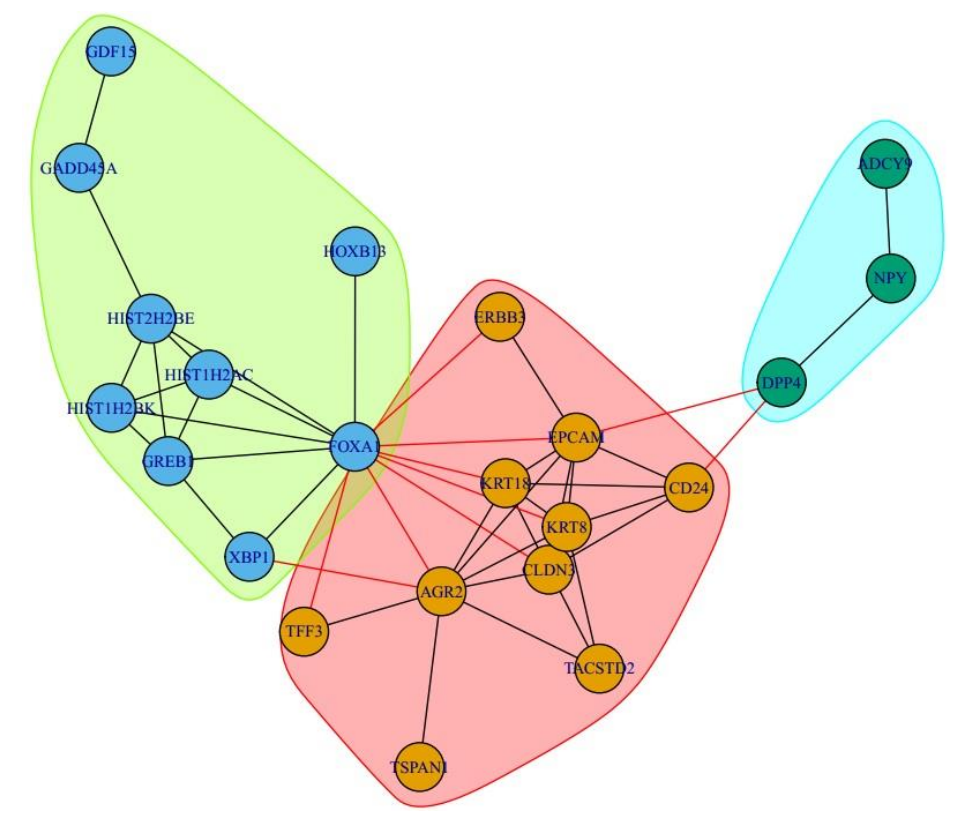

Figure5: Clustering results for GC of the Core network. Nodes in each cluster are situated in the same color aura. First cluster is in red. Second cluster is in green and third cluster with three nodes is in blue.

Apoptosis-related network due to altered Notch3 in ovarian cancer, Signaling Pathways in Glioblastoma, and ERBB3 Signaling Pathway are enriched in Wikipathways containing ERBB3, KRT18, and KRT8 genes. ERBB3 is involved in all 3 pathways. Engagement of this gene in pathways-related cancer (WP2864, WP2261, and WP673) and involvement in the 
biological processes including regulation of cell motility (GO:2000145), regulation of MAPK cascade (GO:0043408), and regulation of signal transduction (GO:0009966) proposes the potential importance of this gene in different cancers. In $2 \mathrm{~B}, \mathrm{CD} 24$ gene has been shown to monopoliz the most GO terms to its self, then the highest number of terms is related to ERBB3 and AGR2. The enrichment analysis for the core network components of 2 and 3 are presented in Supplementary file 4.

In component 2, two genes enriched in Wikipathays. One of these genes, GADD45A, is involved in 12 pathways whose involvement in all of them demonstrates the vital role of this gene in pathogenesis and progression of cancer (TP53 Network WP1742, Imatinib and Chronic Myeloid Leukemia WP3640, ATM Signaling Pathway WP2516, OEndometrial cancer WP4155, G1 to S cell cycle control WP45, Chromosomal and microsatellite instability in colorectal cancer WP4216, and ...). Moreover, this gene is implicated in regulation of apoptotic process (GO:0042981) and activation of MAPKKK activity (GO:0000185) in aspect of biological process in GO. HIST2H2BE and HIST1H2BK genes have monopolized the most GO terms to its self. After them, XBP1 has the highest number of terms. In component 3, ADCY9 has enriched for the most pathways and possesses the most GO terms.

A

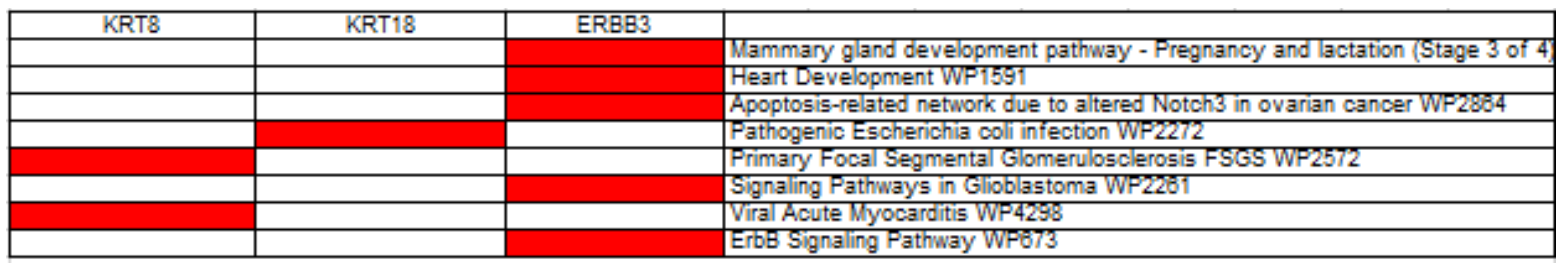

B

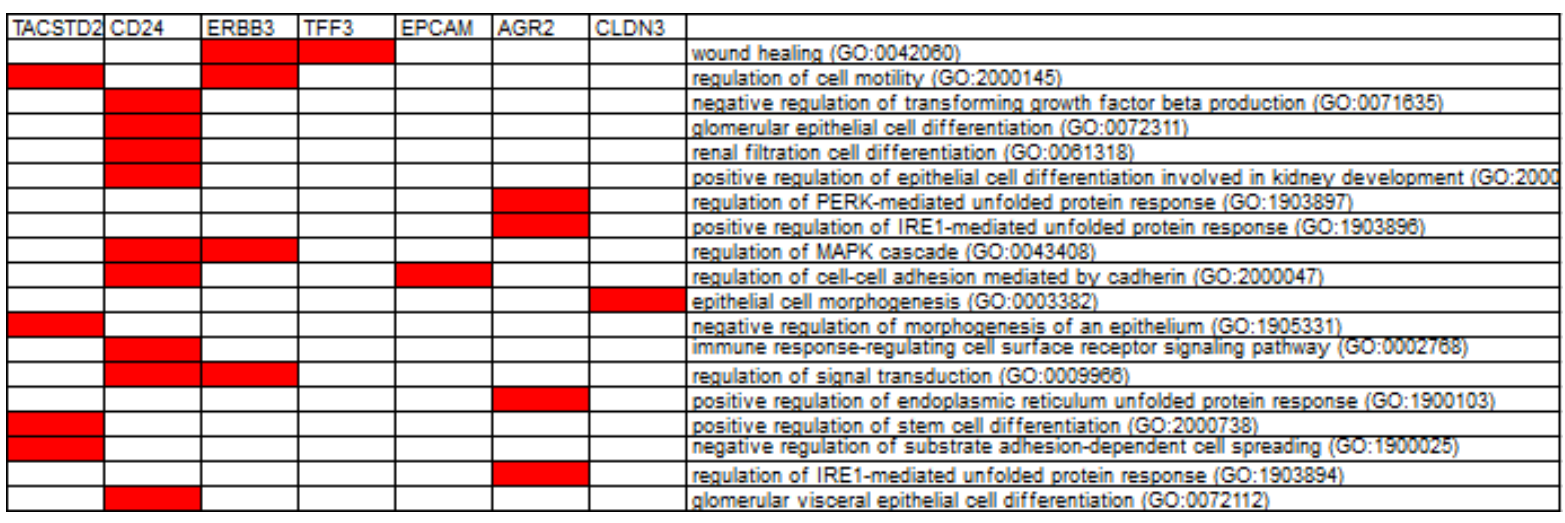

Table1: Enrichment terms for the giant component. 2A illustrates the enriched pathways from WikiPathways signaling datasets. 2B shows the enriched terms for biological process from GO repository. Red cells reveal the involvement of the genes in the enriched elements. 


\section{Discussion}

Integrated bioinformatics analysis mainly focusing on differentially expressed molecule screen has been extensively applied to identify potential biomarkers associated with the diagnosis, treatment, and prognosis of cancers. With two microarray datasets from the GEO database, we identified DEGs in primary PCa and normal tissues. Cell cycle related biological processes are highlighted as the significant items for primary PCa in this study. The association of all of these genes with different cancers has already been confirmed.

FOXA1 one of the core genes has the highest value in both centralities (degree and betweenness) and exhibited upregulation in PvsN analysis. FOXA1 is characterized as a "pioneer factor" having the special property of being able to directly bind DNA (26). Increased expression of FOXA1 has also been observed in colon, lung, thyroid, esophageal and prostate cancer (27). FOXA1 not only plays a role in androgen receptor (AR) signaling, but also regulates the expression of genes engaged in cell cycle regulation in prostate cancer (28-30). Imamura et all. identified IGFBP-3 as a down-stream target of FOXA1 (28). Increased IGFBP-3 expression level following reduction of FOXA1 significantly inhibited proliferation of PCs most likely via the phosphorylation inhibition of signaling mediators in the IGF-1 signaling pathway including MAPK and Akt, and mediated cell cycle arrest through p21 and p27 in PCa cells (28). This gene has been identified as not only an attractive therapeutic target but could potentially function as a biomarker in previous investigations (31).

AGR2, anterior gradient 2, is a member of protein disulfide isomerase (PDI) family resides in the endoplasmic reticulum (ER) (32). AGR2 expression has been found in prostate: primary and metastatic, pancreatic, breast, lung, gastrointestinal and oral solid tumors $(33,34)$. In PvsN analysis, overexprission of this gene has been observed and also had pretty high degree and betweenness centralities. The reduced level of AGR2 expression by using ARG2specific siRNA led to cell cycle arrest at $\mathrm{G}_{0} / \mathrm{G}_{1}$ phase, providing several pieces of evidence that AGR2 is a kind of cell cycle modulator (35). AGR2-silenced cells display significant reduced cell proliferation and invasion of pancreatic cancer cells (36). Arrest in G 0 /G 1 phase of cell cycle due to transient knockdown of AGR2 is correlated with senescence response in malignant prostatic cells (35). Cellular senescence is characterized as a key mechanism of tumor suppression (37). AGR2 has been suggested as a potential biomarker for the diagnosis of some cancers including pituitary adenomas, prostate, human lung adenocarcinoma, and breast (38-41) 
Epithelial cell adhesion molecule (EpCAM), also known as CD326, is a transmembrane glycoprotein acts various roles including cell signalling, migration, proliferation and differentiation besides cell-cell adhesion (42). EpCAM signaling is activated upon regulated intramembrane proteolysis (RIP) and subsequent release of the intracellular domain EpICD, which associates with components of the Wnt pathway (FHL2, $\beta$-catenin, Lef-1), translocates to the nucleus, and functions as transcription regulator. EpCAM induced genes, such as $c$ myc, cyclin D1, mmp7 (43-46), and regulators of the cell cycle and proliferation machinery, in general (47). Overexpression of EpCAM has been found to be associated with enhanced transcription and translation of the proto-oncogene c-myc (43). EpCAM was then found to be expressed at a high level and frequency not only on primary PCa (48) and colon cancer tissues but on most human adenocarcinomas (49) as well as on squamous cell carcinomas (50). In current study, Increased EpCAM expression has been observed and had been identified as a biomarker for hepatocellular carcinoma and prostate cancer $(51,52)$

NPY is a 36 amino-acid sympathetic neurotransmitter which is abundant in the brain (53). NPY has been revealed to be engaged in mitogenic pathways and stimulate cellular proliferation through the Y1-R, its specific G protein-coupled receptor (54). The Y1-R activation is generally associated with decrease of cAMP accumulation, increase of intracellular free calcium concentration $\left(\left[\mathrm{Ca}^{2+}\right]_{\mathrm{i}}\right)$, and modulation of the MAPK pathway via several signaling molecules, including the protein kinase C (PKC) $(54,55)$. Recent researches have shown that NPY can promote cell proliferation of endothelial and increase tumor vascularization through VEGF pathway in some solid tumor cell types $(56,57)$. The mRNA expression level of $N P Y$ is significantly higher in non-aggressive PCa cells than that in aggressive PCa (58). Elevated level of NPY expression has been found in current study and also some studies have found NPY receptors to be overexpressed in neuroblastoma and breast cancers, and emerged as promising targets in cancer diagnosis and therapy (59-61)

CLDN3 belongs to a family of proteins engaged in the formation and function of tight junctions (TJs) (62). Hewitt et al. reported CLDN3, CLDN4, and CLDN7 to be overexpressed in the pancreas, stomach, colon, bladder, ovary, breast, uterus, and in both primary and metastatic prostate cancer and also it has been overexpressed in PvsN analysis (63, 64). Overexpression of $C L D N 3$ is regulated by the EGF-activated downstream pathway, ERK1/2 and PI3K-Akt signaling pathways (65). More importantly, the EGF-induced overexpression of CLDN3 in adenomacarcinoma (ADC) cells reinforces the cell proliferation (65). In another study CLDN3 was suggested to be a potential molecular target for future application in the treatment 
of $\mathrm{ADC}$ (66). It is interesting that almost all of these cell cycle regulatory proteins can be modulated by PI3K signaling (67). ERBB3 can interact with many genetic and signaling pathways, including the PI3K/AKT pathway $(68,69)$. In recent study, G2/M arrest occurred in both MKN45 and SGC7901 by silencing ERBB3. Since G2/M transition is mediated by CDK1, by decreasing cyclinB1, one of its most important regulators, activity of CDK1 was also decreased. Inactivation of Akt by expression of its dominant negative mutant form inhibited cell proliferation by arresting the cells at G2/M phase through decreasing cyclinB1 (70). Overexpression of ERBB3 was reported in different types of cancer including bladder, prostate and breast cancer as well as current study $(71,72)$. In enrichment analysis, ERBB3 is involved in three pathway-related-cancer (WP2864, WP2261, and WP673). This gene is a biomarker for some cancers including oropharyngeal squamous cell carcinoma and pancreatic cancer (73, 74).

GDF15, which encodes growth/differentiation factor-15, is a cytokine and member of the transforming growth factor beta (TGF $\beta$ ) family (75). GDF15 expression is downregulated in metastatic PCa in comparison with the primary tumors, levels of GDF15 RNA and protein are higher in primary prostate tumors (76). In agreement with previous results, elevated GDF15 expression has been found in our PvsN analysis. In prostate cancer, maspin inhibits tumor growth, reduces bone metastasis, and decreases angiogenesis in vitro and in vivo (77-79). Based on the result of an investigation, overexpression of GDF15 blocked maspin gene expression. This finding might explain how overexpression of GDF15 leads to an increased proliferation and invasiveness of prostate carcinoma cells by GDF15 (80). Elevated expression of GDF15 has been found in the samples of patients with breast and colorectal carcinomas (81). In some studies, GDF15 has been identified as diagnostic and prognostic biomarker for prostate, colorectal and ovarian cancer (82-84)

FHL1 is significantly down-regulated expressed and plays important roles in the regulation of development and progression in several types of human tumors including prostatic carcinoma, bladder cancer (BC), breast carcinoma, and prostate and the same result for the current research has been obtained (85-89). FHL1 impacts the progression of cancers by cell cycle regulation of tumors. FHL1 increases the expression of p21 (a CDK inhibitor) through regulation of gene transcription. In addition, p21 plays a role in the maintenance of G2/M-phase arrest, and regulation of cell cycle arrest at the G1/S transition. FHL1 induces the upregulation of p21 and inhibits cyclins and their associated CDKs (mainly cyclin D1), which are the central machinery governing cell cycle progression (90). FHL1 plays important roles not only in activation of the 
tumor suppressor gene $p 21$, but also in repression of the oncogene $c$-myc through interacting with Smad2, Smad3 and Smad4 that are important regulators of cancer development and progression in a casein kinase $1 \delta$-dependent manner (91). It has been emerged as a biomarker for progression of cutaneous squamous cell carcinoma (92).

Growth arrest and DNA-damage-inducible protein 45 alpha (GADD45A) is a downstream target gene of p53 and BRCA1 (breast cancer susceptibility gene 1) (93). GADD45 family involve in cell cycle progression, cell survival and apoptosis $(94,95)$. By interaction with Cdc2 kinase, Gadd45a can dissociate $\mathrm{Cdc} 2 /$ cyclinB 1 complex and mediate $\mathrm{G}_{2} / \mathrm{M}$ cell cycle arrest $(96,97)$. In addition, GADD45a was reported to suppress the tumor angiogenesis through downregulating of VEGFa via blocking the mTOR/STAT3 pathway (98). Downregulation of GADD45 expression can be possible by the activation of several oncogenic signaling molecules, such as c-Myc, $\mathrm{nF \kappa B}$ or Akt (99). GADD45A protein levels were increased significantly in breast cancer (100) compared with normal tissues and also highly expressed in human pancreatic cancer (101), whereas downregulated in bladder cancer tissues (102). In PvsN, decreased expression level of GADD45A was found. Moreover, it has been suggested as a biomarker for PCa (103). In component 2, GADD45A, was enriched in Wikipathays and involved in 12 pathways associated with cancer and its engagement in all of them demonstrates the vital role of this gene in pathogenesis and progression of cancer.

Dipeptidyl peptidase IV (DPPIV), a membrane glycoprotein, controls the activities of mitogenic growth factors and neuropeptides. DPPIV is involved in various biological processes, including cell differentiation, adhesion, immunomodulation, and apoptosis, functions that are critical for the neoplastic transformation regulation (104-106). In prostate cancer cells, overexpression of DPIV is found in benign glands, whereas little or no DPIV is detected in prostate tumor metastases (107). In aspect of expression, the same result has been found in the current study. DPIV up-regulation in prostatic tumors influences tumor growth through two possible mechanisms based on its potential in peptidase activity, that leads to increase cleavage of a propeptide to its active form could stimulate cell proliferation or alternatively DPIV could inactivate a peptide that inhibits cell proliferation (108). DPP4 could be a potential biomarker and target for cancer therapy (109).

In humans, histone H2B is coded by twenty-three different genes, none of which contain introns. All of these genes are located in histone cluster 1 on chromosome 6 and cluster 2 and cluster 3 on chromosome 1 (110). This gene was upregulated in our analysis which is connected to other histone core genes namely HIST1H2BK and HIST1H2AC in Figure 5. This gene also 
is connected to GADD45A a growth arrest gene. A correlation between reduced expression of Gadd45 and increased resistance to topoisomerase I and topoisomerase II inhibitors in a variety of human cell lines was found. Gadd45 could potentially mediate this effect by destabilizing histone-DNA interactions since it was found to interact directly with the four core histones (111). Gadd45 expression was down regulated in our analysis. This downregulation might associated with the HIST2H2BE upreglution but the mechanism behind this process is not documented yet and this needs a further investigation. However, more investigation needs to be conducted on relation of this gene since no study has connected HIST2H2BE to any kind of cancer so far. This gene has also a relatively high betweenness centrality and HIST2H2BE and HIST1H2BK genes have monopolized the most GO terms to its self which are related to the immune response and DNA assembly. Moreover, it can be proposed as a possible diagnosis and prognosis biomarker for PCa.

In conclusion, with the employment of multiple gene expression profile datasets and integrated bioinformatics analysis, we have demonstrated 10 potential biomarkers which have been confirmed by previous investigations. In addition, HIST2H2BE has been identified as a possible biomarker for the pathogenesis of PCa. The results of this study can shed more light on common molecular mechanisms adopted by PCa.

\section{Refrences}

1. Ferlay J, Shin H, Bray F, Forman D, Mathers C, Parkin D. GLOBOCAN 2008 v2. 0, Cancer Incidence and Mortality Worldwide: IARC CancerBase No. 10 [Internet]. Lyon, France: International Agency for Research on Cancer, 2010. Cited 28 Sep 2013. Lung cancer incidence and mortality. 2012. 2. Vance TM, Su J, Fontham ET, Koo SI, Chun OK. Dietary antioxidants and prostate cancer: a review. Nutrition and cancer. 2013;65(6):793-801.

3. Hemminki K, Vaittinen P. Familial breast cancer in the family-cancer database. International journal of cancer. 1998;77(3):386-91.

4. Lichtenstein P, Holm NV, Verkasalo PK, Iliadou A, Kaprio J, Koskenvuo M, et al. Environmental and heritable factors in the causation of cancer-analyses of cohorts of twins from Sweden, Denmark, and Finland. New England journal of medicine. 2000;343(2):78-85.

5. Malumbres M, Barbacid M. To cycle or not to cycle: a critical decision in cancer. Nature Reviews Cancer. 2001;1(3):222-31.

6. Evan GI, Vousden KH. Proliferation, cell cycle and apoptosis in cancer. Nature. 2001;411(6835):342.

7. Schwartz GK, Shah MA. Targeting the cell cycle: a new approach to cancer therapy. Journal of clinical oncology. 2005;23(36):9408-21.

8. Blagden S, Bono Jd. Drugging cell cycle kinases in cancer therapy. Current drug targets. 2005;6(3):325-35.

9. Bjartell A, Montironi R, Berney DM, Egevad L. Tumour markers in prostate cancer II: diagnostic and prognostic cellular biomarkers. Acta Oncologica. 2011;50(sup1):76-84. 
bioRxiv preprint doi: https://doi.org/10.1101/2020.03.05.978205; this version posted March 5, 2020. The copyright holder for this preprint (which was not certified by peer review) is the author/funder. All rights reserved. No reuse allowed without permission.

10. Allison D. Cui X, Page GP, Sabripour M. Microarray data analysis: from disarray to consolidation and consensus Nat Rev Genet. 2006;7:55-65.

11. Wu L, Qu X. Cancer biomarker detection: recent achievements and challenges. Chemical Society Reviews. 2015;44(10):2963-97.

12. Jakupciak JP, Dakubo GD, Maragh S, Parr RL. Analysis of potential cancer biomarkers in mitochondrial DNA. Current opinion in molecular therapeutics. 2006;8(6):500-6.

13. Yang W, Yoshigoe K, Qin X, Liu JS, Yang JY, Niemierko A, et al. Identification of genes and pathways involved in kidney renal clear cell carcinoma. BMC bioinformatics. 2014;15(17):S2.

14. Gautier L, Cope L, Bolstad BM, Irizarry RA. affy - analysis of Affymetrix GeneChip data at the probe level. Bioinformatics. 2004;20(3):307-15.

15. Do JH, Choi D-K. Normalization of microarray data: single-labeled and dual-labeled arrays. Molecules \& Cells (Springer Science \& Business Media BV). 2006;22(3).

16. Ramasamy A, Mondry A, Holmes CC, Altman DG. Key issues in conducting a meta-analysis of gene expression microarray datasets. PLoS medicine. 2008;5(9):e184.

17. Gentleman R, Carey V, Huber W, Hahne F. Genefilter: Methods for filtering genes from microarray experiments. $\mathrm{R}$ package version. 2011;1(0).

18. Smyth GK. Limma: linear models for microarray data. Bioinformatics and computational biology solutions using R and Bioconductor: Springer; 2005. p. 397-420.

19. Luke DA. A user's guide to network analysis in R: Springer; 2015.

20. Langfelder P, Horvath S. WGCNA: an R package for weighted correlation network analysis. BMC bioinformatics. 2008;9(1):559.

21. Csardi G, Nepusz T. The igraph software package for complex network research. InterJournal, Complex Systems. 2006;1695(5):1-9.

22. Piran M, Piran M, Sepahi N, Rahimi A. Systems Biomedicine of Colorectal Cancer Reveals Potential Targets for CRC treatment. bioRxiv. 2020.

23. Kuleshov MV, Jones MR, Rouillard AD, Fernandez NF, Duan Q, Wang Z, et al. Enrichr: a comprehensive gene set enrichment analysis web server 2016 update. Nucleic acids research. 2016;44(W1):W90-W7.

24. Oldham MC, Horvath S, Konopka G, Iwamoto K, Langfelder P, Kato T, et al. Identification and Removal of Outlier Samples Supplement for:" Functional Organization of the Transcriptome in Human Brain. dim (dat1).1(18631):105.

25. Khanin R, Wit E. How scale-free are biological networks. Journal of computational biology. 2006;13(3):810-8.

26. Cirillo LA, Lin FR, Cuesta I, Friedman D, Jarnik M, Zaret KS. Opening of compacted chromatin by early developmental transcription factors HNF3 (FoxA) and GATA-4. Molecular cell. 2002;9(2):279-89.

27. Lin L, Miller CT, Contreras JI, Prescott MS, Dagenais SL, Wu R, et al. The hepatocyte nuclear factor $3 \alpha$ gene, HNF3 $\alpha$ (FOXA1), on chromosome band 14q13 is amplified and overexpressed in esophageal and lung adenocarcinomas. Cancer research. 2002;62(18):5273-9.

28. Imamura Y, Sakamoto S, Endo T, Utsumi T, Fuse M, Suyama T, et al. FOXA1 promotes tumor progression in prostate cancer via the insulin-like growth factor binding protein 3 pathway. PloS one. $2012 ; 7(8)$.

29. Jin H-J, Zhao JC, Ogden I, Bergan RC, Yu J. Androgen receptor-independent function of FoxA1 in prostate cancer metastasis. Cancer research. 2013;73(12):3725-36.

30. Xu Y, Chen S-Y, Ross KN, Balk SP. Androgens induce prostate cancer cell proliferation through mammalian target of rapamycin activation and post-transcriptional increases in cyclin D proteins. Cancer research. 2006;66(15):7783-92.

31. Robinson J, Carroll J. FoxA1 is a key mediator of hormonal response in breast and prostate cancer. Frontiers in endocrinology. 2012;3:68.

32. Chevet E, Fessart D, Delom F, Mulot A, Vojtesek B, Hrstka R, et al. Emerging roles for the pro-oncogenic anterior gradient-2 in cancer development. Oncogene. 2013;32(20):2499-509.

33. Brychtova V, Mohtar A, Vojtesek B, Hupp TR, editors. Mechanisms of anterior gradient-2 regulation and function in cancer. Seminars in cancer biology; 2015: Elsevier.

34. Maresh EL, Mah V, Alavi M, Horvath S, Bagryanova L, Liebeskind ES, et al. Differential expression of anterior gradient gene AGR2 in prostate cancer. BMC cancer. 2010;10(1):680. 
bioRxiv preprint doi: https://doi.org/10.1101/2020.03.05.978205; this version posted March 5, 2020. The copyright holder for this preprint (which was not certified by peer review) is the author/funder. All rights reserved. No reuse allowed without permission.

35. Hu Z, Gu Y, Han B, Zhang J, Li Z, Tian K, et al. Knockdown of AGR2 induces cellular senescence in prostate cancer cells. Carcinogenesis. 2012;33(6):1178-86.

36. Ramachandran V, Arumugam T, Wang H, Logsdon CD. Anterior gradient 2 is expressed and secreted during the development of pancreatic cancer and promotes cancer cell survival. Cancer research. 2008;68(19):7811-8.

37. Campisi J, Di Fagagna FDA. Cellular senescence: when bad things happen to good cells. Nature reviews Molecular cell biology. 2007;8(9):729-40.

38. Tohti M, Li J, Tang C, Wen G, Abdujilil A, Yizim P, et al. Serum AGR2 as a useful biomarker for pituitary adenomas. Clinical neurology and neurosurgery. 2017;154:19-22.

39. Whitaker H, Shiong L, Kay J, Grönberg H, Warren A, Seipel A, et al. N-acetyl-L-aspartyl-Lglutamate peptidase-like 2 is overexpressed in cancer and promotes a pro-migratory and pro-metastatic phenotype. Oncogene. 2014;33(45):5274-87.

40. Chung K, Nishiyama N, Yamano S, Komatsu H, Hanada S, Wei M, et al. Serum AGR2 as an early diagnostic and postoperative prognostic biomarker of human lung adenocarcinoma. Cancer Biomarkers. 2012;10(2):101-7.

41. Salmans ML, Zhao F, Andersen B. The estrogen-regulated anterior gradient 2 (AGR2) protein in breast cancer: a potential drug target and biomarker. Breast Cancer Research. 2013;15(2):204.

42. Ni J, Cozzi PJ, Duan W, Shigdar S, Graham PH, John KH, et al. Role of the EpCAM (CD326) in prostate cancer metastasis and progression. Cancer and Metastasis Reviews. 2012;31(3-4):779-91.

43. Münz M, Kieu C, Mack B, Schmitt B, Zeidler R, Gires O. The carcinoma-associated antigen EpCAM upregulates c-myc and induces cell proliferation. Oncogene. 2004;23(34):5748-58.

44. Maetzel D, Denzel S, Mack B, Canis M, Went P, Benk M, et al. Nuclear signalling by tumourassociated antigen EpCAM. Nature cell biology. 2009;11(2):162-71.

45. Denzel S, Maetzel D, Mack B, Eggert C, Bärr G, Gires O. Initial activation of EpCAM cleavage via cell-to-cell contact. BMC cancer. 2009;9(1):402.

46. Chaves-Perez A, Mack B, Maetzel D, Kremling H, Eggert C, Harreus U, et al. EpCAM regulates cell cycle progression via control of cyclin D1 expression. Oncogene. 2013;32(5):641-50.

47. Maaser K, Borlak J. A genome-wide expression analysis identifies a network of EpCAMinduced cell cycle regulators. British journal of cancer. 2008;99(10):1635-43.

48. Ni J, Cozzi P, Hao J, Beretov J, Chang L, Duan W, et al. Epithelial cell adhesion molecule (EpCAM) is associated with prostate cancer metastasis and chemo/radioresistance via the PI3K/Akt/mTOR signaling pathway. The international journal of biochemistry \& cell biology. 2013;45(12):2736-48.

49. Went PT, Lugli A, Meier S, Bundi M, Mirlacher M, Sauter G, et al. Frequent EpCam protein expression in human carcinomas. Human pathology. 2004;35(1):122-8.

50. Quak J, Van Dongen G, Brakkee J, Hayashida D, Balm A, Snow G, et al. Production of a monoclonal antibody (K 931) to a squamous cell carcinoma associated antigen identified as the 17-1A antigen. Hybridoma. 1990;9(4):377-87.

51. Schulze K, Gasch C, Staufer K, Nashan B, Lohse AW, Pantel K, et al. Presence of EpCAMpositive circulating tumor cells as biomarker for systemic disease strongly correlates to survival in patients with hepatocellular carcinoma. International journal of cancer. 2013;133(9):2165-71.

52. Danila DC, Fleisher M, Scher HI. Circulating tumor cells as biomarkers in prostate cancer. Clinical Cancer Research. 2011;17(12):3903-12.

53. ZUKOWSKA-GROJEC Z. Neuropeptide Y: a novel sympathetic stress hormone and more. Annals of the new York Academy of Sciences. 1995;771(1):219-33.

54. MANNON PJ, MELE JM. Peptide YY Y1 receptor activates mitogen-activated protein kinase and proliferation in gut epithelial cells via the epidermal growth factor receptor. Biochemical Journal. 2000;350(3):655-61.

55. Pedrazzini T, Pralong F, Grouzmann E. Neuropeptide Y: the universal soldier. Cellular and Molecular Life Sciences CMLS. 2003;60(2):350-77.

56. Zukowska-Grojec Z, Karwatowska-Prokopczuk E, Fisher TA, Ji H. Mechanisms of vascular growth-promoting effects of neuropeptide $\mathrm{Y}$ : role of its inducible receptors. Regulatory peptides. 1998;75:231-8.

57. Kitlinska J, Abe K, Kuo L, Pons J, Yu M, Li L, et al. Differential effects of neuropeptide Y on the growth and vascularization of neural crest-derived tumors. Cancer research. 2005;65(5):1719-28. 
bioRxiv preprint doi: https://doi.org/10.1101/2020.03.05.978205; this version posted March 5, 2020. The copyright holder for this preprint (which was not certified by peer review) is the author/funder. All rights reserved. No reuse allowed without permission.

58. Liu A-j, Furusato B, Ravindranath L, Chen Y-m, Srikantan V, Mcleod DG, et al. Quantitative analysis of a panel of gene expression in prostate cancer - with emphasis on NPY expression analysis. Journal of Zhejiang University SCIENCE B. 2007;8(12):853-9.

59. Czarnecka M, Trinh E, Lu C, Kuan-Celarier A, Galli S, Hong S-H, et al. Neuropeptide Y receptor Y5 as an inducible pro-survival factor in neuroblastoma: implications for tumor chemoresistance. Oncogene. 2015;34(24):3131-43.

60. Medeiros PJ, Jackson DN. Neuropeptide Y Y5-receptor activation on breast cancer cells acts as a paracrine system that stimulates VEGF expression and secretion to promote angiogenesis. Peptides. 2013;48:106-13.

61. Li J, Tian Y, Wu A. Neuropeptide Y receptors: a promising target for cancer imaging and therapy. Regenerative biomaterials. 2015;2(3):215-9.

62. Latorre IJ, Frese KK, Javier RT. Tight junction proteins and cancer. Tight Junctions: Springer; 2006. p. 116-34.

63. Hewitt KJ, Agarwal R, Morin PJ. The claudin gene family: expression in normal and neoplastic tissues. BMC cancer. 2006;6(1):186.

64. Bartholow TL, Chandran UR, Becich MJ, Parwani AV. Immunohistochemical profiles of claudin-3 in primary and metastatic prostatic adenocarcinoma. Diagnostic pathology. 2011;6(1):12.

65. de Souza WF, Fortunato-Miranda N, Robbs BK, de Araujo WM, de-Freitas-Junior JC, Bastos LG, et al. Claudin-3 overexpression increases the malignant potential of colorectal cancer cells: roles of ERK1/2 and PI3K-Akt as modulators of EGFR signaling. PloS one. 2013;8(9).

66. Zhang L, Wang Y, Zhang B, Zhang H, Zhou M, Wei M, et al. Claudin-3 expression increases the malignant potential of lung adenocarcinoma cells: role of epidermal growth factor receptor activation. Oncotarget. 2017;8(14):23033.

67. Zhang X, Tang N, Hadden TJ, Rishi AK. Akt, FoxO and regulation of apoptosis. Biochimica et Biophysica Acta (BBA)-Molecular Cell Research. 2011;1813(11):1978-86.

68. Sithanandam G, Smith GT, Masuda A, Takahashi T, Anderson LM, Fornwald LW. Cell cycle activation in lung adenocarcinoma cells by the ErbB3/phosphatidylinositol 3-kinase/Akt pathway. Carcinogenesis. 2003;24(10):1581-92.

69. Sithanandam G, Anderson L. The ERBB3 receptor in cancer and cancer gene therapy. Cancer gene therapy. 2008;15(7):413-48.

70. Choi YH, Zhang L, Lee W, Park K. Genistein-induced G2/M arrest is associated with the inhibition of cyclin B1 and the induction of p21 in human breast carcinoma cells. International journal of oncology. 1998;13(2):391-7.

71. Howe LR, Brown PH. Targeting the HER/EGFR/ErbB family to prevent breast cancer. Cancer prevention research. 2011;4(8):1149-57.

72. K Jathal M, Chen L, Mudryj M, M Ghosh P. Targeting ErbB3: the new RTK (id) on the prostate cancer block. Immunology, Endocrine \& Metabolic Agents in Medicinal Chemistry (Formerly Current Medicinal Chemistry-Immunology, Endocrine and Metabolic Agents). 2011;11(2):131-49.

73. Qian G, Jiang N, Wang D, Newman S, Kim S, Chen Z, et al. Heregulin and HER3 are prognostic biomarkers in oropharyngeal squamous cell carcinoma. Cancer. 2015;121(20):3600-11.

74. Thomas G, Chardès T, Gaborit N, Mollevi C, Leconet W, Robert B, et al. HER3 as biomarker and therapeutic target in pancreatic cancer: new insights in pertuzumab therapy in preclinical models. Oncotarget. 2014;5(16):7138.

75. Vaňhara P, Hampl A, Kozubik A, Souček K. Growth/differentiation factor-15: prostate cancer suppressor or promoter? Prostate cancer and prostatic diseases. 2012;15(4):320-8.

76. Rasiah KK, Kench JG, Gardiner-Garden M, Biankin AV, Golovsky D, Brenner PC, et al. Aberrant neuropeptide $\mathrm{Y}$ and macrophage inhibitory cytokine-1 expression are early events in prostate cancer development and are associated with poor prognosis. Cancer Epidemiology and Prevention Biomarkers. 2006;15(4):711-6.

77. Cher ML, Biliran HR, Bhagat S, Meng Y, Che M, Lockett J, et al. Maspin expression inhibits osteolysis, tumor growth, and angiogenesis in a model of prostate cancer bone metastasis. Proceedings of the National Academy of Sciences. 2003;100(13):7847-52.

78. Watanabe M, Nasu Y, Kashiwakura Y, Kusumi N, Tamayose K, Nagai A, et al. Adenoassociated virus 2-mediated intratumoral prostate cancer gene therapy: long-term maspin expression efficiently suppresses tumor growth. Human gene therapy. 2005;16(6):699-710. 
bioRxiv preprint doi: https://doi.org/10.1101/2020.03.05.978205; this version posted March 5, 2020. The copyright holder for this preprint (which was not certified by peer review) is the author/funder. All rights reserved. No reuse allowed without permission.

79. Hall DC, Johnson-Pais TL, Grubbs B, Bernal R, Leach RJ, Padalecki SS, editors. Maspin reduces prostate cancer metastasis to bone. Urologic Oncology: Seminars and Original Investigations; 2008: Elsevier.

80. Tsui K-H, Chang Y-L, Feng T-H, Chung L-C, Lee T-Y, Chang P-L, et al. Growth differentiation factor-15 upregulates interleukin-6 to promote tumorigenesis of prostate carcinoma PC3 cells. Journal of molecular endocrinology. 2012;49(2):153.

81. Welch JS, Ricote M, Akiyama TE, Gonzalez FJ, Glass CK. PPAR $\gamma$ and PPAR $\delta$ negatively regulate specific subsets of lipopolysaccharide and IFN- $\gamma$ target genes in macrophages. Proceedings of the National Academy of Sciences. 2003;100(11):6712-7.

82. Li C, Wang X, Casal I, Wang J, Li P, Zhang W, et al. Growth differentiation factor 15 is a promising diagnostic and prognostic biomarker in colorectal cancer. Journal of cellular and molecular medicine. 2016;20(8):1420-6.

83. Staff AC, Bock AJ, Becker C, Kempf T, Wollert KC, Davidson B. Growth differentiation factor-15 as a prognostic biomarker in ovarian cancer. Gynecologic oncology. 2010;118(3):237-43.

84. Winand FJ, Boegemann M, Gallitz I, Hertle L, Semjonow A, Eveslage M, et al. GDF15 and Hepcidin as prognostic factors in patients with prostate Cancer. Journal of Molecular Biomarkers \& Diagnosis. 2014;5(6):1.

85. Shen Y, Jia Z, Nagele RG, Ichikawa H, Goldberg GS. SRC uses Cas to suppress Fhl1 in order to promote nonanchored growth and migration of tumor cells. Cancer Research. 2006;66(3):1543-52.

86. Sakashita K, Mimori K, Tanaka F, Kamohara Y, Inoue H, Sawada T, et al. Clinical significance of loss of Fhl1 expression in human gastric cancer. Annals of surgical oncology. 2008;15(8):2293.

87. Matsumoto M, Kawakami K, Enokida H, Toki K, Matsuda R, Chiyomaru T, et al. CpG hypermethylation of human four-and-a-half LIM domains 1 contributes to migration and invasion activity of human bladder cancer. International journal of molecular medicine. 2010;26(2):241-7.

88. Niu C, Liang C, Guo J, Cheng L, Zhang H, Qin X, et al. Downregulation and growth inhibitory role of FHL1 in lung cancer. International journal of cancer. 2012;130(11):2549-56.

89. Koike K, Kasamatsu A, Iyoda M, Saito Y, Kouzu Y, Koike H, et al. High prevalence of epigenetic inactivation of the human four and a half LIM domains 1 gene in human oral cancer. International journal of oncology. 2013;42(1):141-50.

90. Ding L, Niu C, Zheng Y, Xiong Z, Liu Y, Lin J, et al. FHL1 interacts with oestrogen receptors and regulates breast cancer cell growth. Journal of cellular and molecular medicine. 2011;15(1):72-85. 91. Ding L, Wang Z, Yan J, Yang X, Liu A, Qiu W, et al. Human four-and-a-half LIM family members suppress tumor cell growth through a TGF- $\beta$-like signaling pathway. The Journal of clinical investigation. 2009;119(2):349-61.

92. Riihilä PM, Nissinen LM, Ala-Aho R, Kallajoki M, Grénman R, Meri S, et al. Complement factor $\mathrm{H}$ : a biomarker for progression of cutaneous squamous cell carcinoma. Journal of Investigative Dermatology. 2014;134(2):498-506.

93. Ishiguro H, Kimura M, Funahashi H, Ogawa R, Shiozaki M, Shibata T, et al. Tu1926 Growth Arrest and DNA Damage Inducible Protein 45 Alpha (GADD45A) Expression Is Involved in Patient Prognosis in Esophageal Cancer. Gastroenterology. 2013;144(5):S-882.

94. Cretu A, Sha X, Tront J, Hoffman B, Liebermann DA. Stress sensor Gadd45 genes as therapeutic targets in cancer. Cancer therapy. 2009;7(A):268.

95. Takekawa M, Saito H. A family of stress-inducible GADD45-like proteins mediate activation of the stress-responsive MTK1/MEKK4 MAPKKK. Cell. 1998;95(4):521-30.

96. Sheng Z, Sun W, Smith E, Cohen C, Sheng Z, Xu X-X. Restoration of positioning control following Disabled-2 expression in ovarian and breast tumor cells. Oncogene. 2000;19(42):4847-54.

97. Jin S, Tong T, Fan W, Fan F, Antinore MJ, Zhu X, et al. GADD45-induced cell cycle G2-M arrest associates with altered subcellular distribution of cyclin B1 and is independent of p38 kinase activity. Oncogene. 2002;21(57):8696-704.

98. Yang F, Zhang W, Li D, Zhan Q. Gadd45a suppresses tumor angiogenesis via inhibition of the mTOR/STAT3 protein pathway. Journal of Biological Chemistry. 2013;288(9):6552-60.

99. Siafakas AR, Richardson DR. Growth arrest and DNA damage-45 alpha (GADD45 $\alpha$ ). The international journal of biochemistry \& cell biology. 2009;41(5):986-9.

100. Tront JS, Willis A, Huang Y, Hoffman B, Liebermann DA. Gadd45a levels in human breast cancer are hormone receptor dependent. Journal of translational medicine. 2013;11(1):131. 
bioRxiv preprint doi: https://doi.org/10.1101/2020.03.05.978205; this version posted March 5, 2020. The copyright holder for this preprint (which was not certified by peer review) is the author/funder. All rights reserved. No reuse allowed without permission.

101. Dong M, Zhou J, Kong F, Guo K, Tian Y, Dong Y. Expressions of p53 and Gadd45a proteins in human pancreatic cancer and their clinicopathological significance. Zhongguo yi xue ke xue yuan xue bao Acta Academiae Medicinae Sinicae. 2005;27(5):628-32.

102. Han N, Yuan F, Xian P, Liu N, Liu J, Zhang H, et al. GADD45a Mediated Cell Cycle Inhibition Is Regulated By P53 In Bladder Cancer. OncoTargets and therapy. 2019;12:7591.

103. Reis I, Ramachandran K, Speer C, Gordian E, Singal R. Serum GADD45a methylation is a useful biomarker to distinguish benign vs malignant prostate disease. British journal of cancer. 2015;113(3):460-8.

104. Boonacker E, Van Noorden CJ. The multifunctional or moonlighting protein CD26/DPPIV. European journal of cell biology. 2003;82(2):53-73.

105. Proost P, Struyf S, Schols D, Opdenakker G, Sozzani S, Allavena P, et al. Truncation of macrophage-derived chemokine by CD26/dipeptidyl-peptidase IV beyond its predicted cleavage site affects chemotactic activity and CC chemokine receptor 4 interaction. Journal of Biological Chemistry. 1999;274(7):3988-93.

106. Bauvois B. A collagen-binding glycoprotein on the surface of mouse fibroblasts is identified as dipeptidyl peptidase IV. Biochemical Journal. 1988;252(3):723-31.

107. Piazza GA, Callanan HM, Mowery J, Hixson D. Evidence for a role of dipeptidyl peptidase IV in fibronectin-mediated interactions of hepatocytes with extracellular matrix. Biochemical Journal. 1989;262(1):327-34.

108. Nanus DM. Of peptides and peptidases: the role of cell surface peptidases in cancer. AACR; 2003.

109. Enz N, Vliegen G, De Meester I, Jungraithmayr W. CD26/DPP4-a potential biomarker and target for cancer therapy. Pharmacology \& therapeutics. 2019;198:135-59.

110. Molden RC, Bhanu NV, LeRoy G, Arnaudo AM, Garcia BA. Multi-faceted quantitative proteomics analysis of histone $\mathrm{H} 2 \mathrm{~B}$ isoforms and their modifications. Epigenetics Chromatin. 2015;8:15.

111. Carrier F, Georgel PT, Pourquier P, Blake M, Kontny HU, Antinore MJ, et al. Gadd45, a p53responsive stress protein, modifies DNA accessibility on damaged chromatin. Molecular and cellular biology. 1999;19(3):1673-85. 